\title{
THE POWER OF HABITS: EVALUATION OF A MOBILE HEALTH SOLUTION FOR THE MANAGEMENT OF NARCOLEPSY
}

\author{
Staszak, Wiktoria; \\ de Paula, Danielly; \\ Uebernickel, Falk \\ Hasso Plattner Institute
}

\begin{abstract}
Mobile health, or mHealth, solutions offer great potential in the area of self-monitoring of chronic conditions, where most of the day-to-day management of the condition is done at home by the patient or their caregivers. Narcolepsy is a chronic sleep condition caused by an orexin deficiency in the brain resulting in its inability to regulate sleep cycles, causing poor quality sleep during the day and problems with wakefulness during the day. This paper set out to investigate whether Habitual, an app for tracking symptoms, daily habits and medication adherence for people with narcolepsy could increase their sense of empowerment. Ten participants were asked to test the app during a period of 30 days, after which they were asked to answer a survey to investigate whether their perception of their empowerment towards the management of narcolepsy had changed. Although using the app for only 30 days provides a very limited understanding of the impact of Habitual, this study shows positive indication for future mHealth solutions for the management of narcolepsy. Future studies should test the openness to using an app for the management of narcolepsy with a wider cohort.
\end{abstract}

Keywords: User centred design, Early design phases, Evaluation

\author{
Contact: \\ Staszak, Wiktoria \\ Hasso Plattner Institute \\ Chair of Design Thinking and Innovation Research \\ Switzerland \\ wiktoria.staszak@student.hpi.de
}

Cite this article: Staszak, W., de Paula, D., Uebernickel, F. (2021) 'The Power of Habits: Evaluation of a Mobile Health Solution for the Management of Narcolepsy', in Proceedings of the International Conference on Engineering Design (ICED21), Gothenburg, Sweden, 16-20 August 2021. DOI:10.1017/pds.2021.569 


\section{INTRODUCTION}

Mobile health, or mHealth, solutions offer great potential in the area of self-monitoring of chronic conditions, where most of the day-to-day management of the condition is undertaken at home by the patient or their caregivers. One of the aims of MHealth solutions is to empower the patient by helping them to understand and actively participate in the decisions regarding their health. This is particularly important for rare diseases, where patients come into contact with medical personnel who are not familiar with their particular condition. One example is narcolepsy, which lies at the heart of this study.

Narcolepsy is a rare chronic sleep condition which is generally caused by a hypocretin deficiency in the brain resulting in its inability to regulate sleep cycles, causing poor quality sleep at night and problems with wakefulness during the day (Dauvilliers et al., 2007). Apart from nocturnal sleep disruption and excessive daytime sleepiness (EDS), people with narcolepsy experience a broad range of other symptoms, such as cognitive difficulties, vivid dreams and cataplexy (in the case of narcolepsy type 1). Narcolepsy is managed through a combination of pharmacological and non-pharmacological treatments. There currently is no tool that allows of persons with narcolepsy $(\mathrm{PwN})$ to track their symptoms, medication and daily habits, and the impact the condition has on quality of life. It is therefore more difficult for PwN to understand how a change in daily habits can affect their symptoms. Previous solutions for the tracking of narcolepsy symptoms have focused on capturing the burden of symptoms, without considering the impact of daily habits (Quaedackers et al., 2020). To address this gap, our study aims to measure whether Habitual — an app that measures symptoms, medication adherence and daily habitscan increase a PwN's sense of empowerment by helping them to manage the condition. Two research questions guide this study:

- $\quad$ RQ1: Does Habitual - a symptom tracking app for people with narcolepsy - have an impact on how participants understand their condition and and on their perceived sense of empowerment?

- RQ2: What are the relevant features to consider when designing mHealth solutions?

To answer these RQs, the study adopts a user-centred approach (Uebernickel et al., 2020) to test an existing prototype app called Habitual. Ten participants were asked to test the app during a period of 30 days, after which they were asked to answer a survey to investigate their perception of its usefulness (Davis, 1989) and whether they felt more empowered (Cerezo et al., 2016) in terms of the management of their narcolepsy condition as a result. The study intends to contribute to academia by advancing the knowledge of how user-centred approaches can be used to develop mHealth interventions for people with narcolepsy, and mHealth solutions more generally.

\section{RELATED WORK}

This section presents the theoretical background relating to mHealth solutions in the area of narcolepsy, followed by previous work on digital companions for symptom monitoring for narcolepsy, on related technology acceptance models, and on measurement of patient empowerment.

\subsection{The importance and challenges of mHealth application in monitoring narcolepsy symptoms}

The World Health Organization (WHO) define mHealth, also known as Mobile Health as "medical and public health practice supported by mobile devices, such as mobile phones, patient monitoring devices, personal digital assistants (PDAs), and other wireless devices" (World Health Organization, 2011). Some examples of applications of mHealth include the improvement of treatment adherence by supporting behaviour change, and applications that act as digital therapeutics (Rowland et al., 2020). Within the mHealth context, one relevant area is the management of the rare chronic disease, narcolepsy. People with narcolepsy experience a wide range of symptoms, such as cognitive difficulties, brain fog and memory problems, which heavily impact their quality of life (Raggi et al., 2019). The severity of symptoms as well as their impact on the daily life of the affected person are normally measured through patient self-reporting captured by using questionnaires. However, a relatively recent study found that currently available questionnaires - e.g. Epworth Sleepiness Scale (ESS), the Swiss Narcolepsy Scale (SNS) - do not capture the patient's individual narcolepsy experience, and their unique set of symptoms and impact on daily life, well enough (Kallweit et al., 2017). One solution aimed at filling this gap is the Narcolepsy monitor developed by Quaedackers and colleagues (Quaedackers et al., 2020). The narcolepsy monitor aims to offer people with narcolepsy a convenient 
way to report the burden of their symptoms. However, the app focuses on measuring the impact of symptoms on the day-to-day functioning of patients, rather than reporting the severity and frequency of their occurrence. Seven patients had been recruited to try out the application. Despite the app receiving excellent ratings overall, patients stated that they would have liked to be able to capture the daily variation of the severity of their symptoms, as well as visualize the resulting data. Furthermore the app fails to link behaviours to symptoms (Quaedackers et al., 2020).

Despite the large increase in the overall use of mHealth apps, and the recognition of their potential in the monitoring of health, especially of chronic conditions, their success in helping achieve health-related objectives is, ultimately, highly dependent upon the user experience of the system.

\subsection{Technology Acceptance Models: constructs for mHealth application to monitor narcolepsy symptoms}

The poor adherence to the long-term use of mHealth interventions has placed greater emphasis on the design and overall usability of the system. One theory, which aims to understand why users accept or reject a technology, is the Technology Acceptance Model (TAM) by Davis (Davis, 1989). According to TAM, user acceptance of a technology is influenced by their perception of its usefulness and of its ease of use (Davis, 1989). Perceived usefulness refers to the extent to which users believe the technology helps them with carrying out a specific task, while the perceived ease of use is "the degree to which a person believes that using a particular system would be free of effort" (Davis, 1989). These two components affect users' attitude towards a technology and directly influence their propensity to utilize a particular technology in their everyday lives. Therefore, by developing solutions which address an unmet need of a user in an intuitive manner, it is possible to increase the adoption of mHealth solutions. The Unified Theory of Acceptance and Use of Technology (UTAUT) by Venkatesh (Venkatesh et al., 2012), expands the TAM by considering two additional factors: social influence, and facilitating conditions. Social influence refers to the weight of importance given to the belief of others that one should use the technology. Facilitating conditions refer to the belief that one has the required infrastructure needed to carry out the task. UTAUT2 is an extended version of UTAUT that includes three further factors that influence acceptance: hedonic motivation, habit, and price value (Venkatesh et al., 2012). Hedonic motivation refers to the pleasure derived from using the system, while habit captures the automatic behaviour involved in using a technology that is developed over time, and cost and pricing structure reflect the relative cost that the user has to bear. This differs from the UTAUT insofar as in the organizational context, the end-user of the system is usually not the bearer of the cost. A recent review found that most studies that used TAM and UTAUT evaluated finished products. However, the authors pointed out that using these models to assess product concepts can make it easier to integrate the necessary changes (Acosta Salgado et al., 2020).

\subsection{The importance of patient empowerment}

The topic of patient empowerment has gained popularity since 2012 when the World Health Organization set it as one of its strategic priority areas in its policy for health and well-being to be achieved as part of its Health 2020 project (World Health Organization Regional Office for Europe, 2012). Although the literature abounds with definitions, a detailed definition of patient empowerment is offered by Small et al. (2013), who describe it as "[a] process and outcome arising from communication between health care professionals and patients involving the sharing of resources over information relating to illness, which enhances the patient's feelings of control, self-efficacy, coping abilities and ability to effect change over their condition" (Small et al., 2013).

Patient empowerment is especially important in the area of rare diseases, where the low prevalence of the condition means that the day-to-day management of the condition frequently involves healthcare professionals who have little knowledge of the condition. The empowerment of patients with rare conditions, such as narcolepsy, relies on patients' ability to work better together with healthcare professionals, and therefore help make the best treatment decisions by providing a more in-depth level of education and a deeper level of involvement in the decision making process (Holmström and Röing, 2010).

According to a review of patient empowerment, the most commonly mentioned components of patient empowerment are: participation in decision making, gaining control, knowledge acquisition, coping skills, positive attitude towards disease management, increasing the sense of meaning to patients' 
experience with disease, motivation, trust, self-care, and sharing and capacity building (Cerezo et al., 2016).

\section{METHODOLOGY}

\subsection{Design of Habitual}

Habitual was developed as a result of a design project in collaboration between two universities. In total, six students from both universities worked together on the challenge of 'How could we support people living with narcolepsy on their whole patient journey?'. The project set out to identify an unmet need faced by people with narcolepsy and to develop a solution to address it. It was carried out over a period of 9 months and followed the Design Thinking methodology (Uebernickel et al., 2020). Design Thinking is a well-know human-centred design approach that has been applied repeatedly in the context of healthcare (De Paula et al., 2021).

Over the 9 months of the project, the needs of narcolepsy patients were extensively researched, including in the form of interviews, and several prototypes were created. After several rounds of scoping interviews, the tracking of symptoms and habits for the effective monitoring of the condition emerged as an, as yet, unmet need. Due to the high level of day-to-day variability in the severity of symptoms, for most individuals the tracking of symptoms, as well as daily behaviours that may influence the severity of symptoms (e.g. poor sleep hygiene), was identified as important for self-monitoring of the condition. Habitual was developed to address that need. Following the successful implementation of the first iteration of Habitual, as well as positive feedback gathered through 1:1 testing sessions with potential end-users, a pilot-study was carried out to investigate the impact of Habitual on the feeling of empowerment of the user. In the next section, we explain how the study was conducted.

\subsection{Study Procedure}

First, participants were recruited in August and September 2020 with the help of the patient organization Wake Up Narcolepsy. A brochure outlining the study was published on the Wake Up Narcolepsy website and social media channels. The inclusion criteria for this study were, at least 18 years of age, diagnosis of Type 1 narcolepsy and access to a device with internet access. Out of the 13 participants who registered, 10 successfully completed the study. The cohort consisted of 9 females and 1 male, of ages between 22-56 (mean =39.4), diagnosed between 2007 and 2019. Nine of the participants were from the United States, one from Canada. Participants were then sent the link to access the app and were asked to use it twice a day for 30 days to track their symptoms and behaviours. After the 30-day period, participants were asked to answer a survey (see Table 1) aimed to investigate the impact of Habitual on the participants' perception of empowerment. The survey items were constructed based on existing empowerment measurements (Cerezo et al., 2016). The final part of the survey asked whether the participant would be willing to take part in an online interview to talk about their experience with Habitual. Out of the ten participants, three agreed to being interviewed, and one provided answers to the questions in a written format. Finally, the interviews took place within a week of the completion of the survey and were carried out online through Zoom. The interview guide covered (i) experiences when using Habitual, (ii) feedback on the existing features, and (iii) ideas for functionality to be added to future versions of Habitual. The average interview time was 28 minutes. The interviews were recorded and transcribed automatically using OtterAI transcription service. The interviewees were coded into: P04782, P30922, and P83277.

\subsection{Data Analysis}

The survey data was extracted from SurveyMonkey and analysed using Python. The Likert scale was converted to numerical values between 0 and 4, where 4 is "Strongly Agree" and 0 is "Strongly disagree". Figure 2 presents the impact of Habitual on the individual empowerment based on existing components of patient empowerment (Cerezo et al., 2016). The interview transcripts were analysed according to a rigorous methodology for qualitative analysis (Gioia et al., 2012). Two researchers conducted the coding of the interviews using the open coding technique and then compared results. In case of discrepancies, a third researcher was consulted. The coding was done using the qualitative data analysis software ATLAS.ti. The coding of the interviews resulted in 45 first order codes from which 
Table 1. Overview of the Measures of empowerment for narcolepsy based on Cerezo et al. (2016)

\begin{tabular}{|c|l|}
\hline Participant empowerment metrics & Survey Item \\
\hline Knowledge acquisition & I notice more how my symptoms change over time. \\
I am more aware of patterns in my symptoms. \\
I better understand why my symptoms change. \\
I feel more confident making minor changes to my treatment \\
(e.e time at which medication is taken) because I understand \\
how it impacts my symptoms. \\
I am more aware which symptoms that I am experiencing are \\
caused by narcolepsy, and which aren't.
\end{tabular}

4 second order codes or categories were deducted. The four categories resulting from the analysis were suggestions for additional functionality, ways that Habitual impacted the empowerment, motivation to use the app, and challenges due to narcolepsy. Examples for each of the second level categories can be found in Table 2.

\section{RESULTS}

\subsection{About Habitual}

The Habitual app enables the tracking of symptoms through short questionnaires which can be answered at various points in the day, such as a set of questions about the nocturnal sleep which is to be answered in the morning (see Figure 1b). Since narcolepsy symptoms do not only impact the physical wellbeing but also the day-to-day function of the person, the app includes questions about the impact of narcolepsy on areas like work attitudes (productivity, motivation) as well as social interactions. The captured datapoints are visualized through a series of graphs, arranged into various information granularity levels, ranging from indices combining various categories of questions (e.g. all sleep related questions) to individual questions. The goal of the indices is to capture the overall impact and hence enable the comparison of data over time. Allowing the user to explore the graph, aims to help with better disease understanding and therefore has advantages over filling out paper-based forms. Habitual aims to empower its users not only through tracking and visualization of symptoms and their impact, but also provide educational resources in the form of short articles related to the behavioural management of narcolepsy such as sleep hygiene (Figure 1c). Habitual is a web app which participants can access through a link which was provided at the beginning of the study. Although the web application hosted on the Heroku cloud hosting service, all data including usage data was stored locally in the browser using IndexDB technology.

\subsection{Evaluation of Habitual}

\subsubsection{Effect of Habitual on empowerment}

As previously mentioned the impact of Habitual was determined by measurements of empowerment as recommended by researchers (Cerezo et al., 2016). The survey aimed to measure whether Habitual has changed the participant's perception of their condition, and therefore determine if Habitual was 
Table 2. Examples of coding categories resulting from the interviews

\begin{tabular}{|c|c|c|}
\hline Category & Code & Quote \\
\hline \multirow[t]{2}{*}{$\begin{array}{l}\text { Effect of Habitual on } \\
\text { empowerment }\end{array}$} & Help communicate symptoms & $\begin{array}{l}\text { "it helps us have a much better conversa- } \\
\text { tion with our doctors." } P 83277\end{array}$ \\
\hline & Increase symptom awareness & $\begin{array}{l}\text { "It really made me think about things that } \\
\text { I hadn't. And I've been a narcoleptic for } \\
\text { a very, very long time." } P 04782\end{array}$ \\
\hline \multirow[t]{4}{*}{$\begin{array}{l}\text { Suggestions for } \\
\text { additional } \\
\text { functionality }\end{array}$} & Anchoring it with existing habits & $\begin{array}{l}\text { "it was a little more difficult at night (...) } \\
\text { because I would fall asleep. And then } \\
\text { after waking up I would kind of forget to } \\
\text { to log it, but I tried to anchor it with when } \\
\text { I ate dinner" } P 83277\end{array}$ \\
\hline & Reminders/ Notifications & $\begin{array}{l}\text { "if it had reminders, that would have been } \\
\text { very, very helpful." P30922 }\end{array}$ \\
\hline & Visualization & $\begin{array}{l}\text { "I think it would be really cool to be able } \\
\text { to see day to day, but then to see what } \\
\text { your month looks like, and to see how } \\
\text { the pattern changes, when the days get } \\
\text { shorter." P30922 }\end{array}$ \\
\hline & Personalization & $\begin{array}{l}\text { "Have it asking questions about the symp- } \\
\text { toms that are especially pertinent to like, } \\
\text { each person's experience ." P30922 }\end{array}$ \\
\hline \multirow[t]{2}{*}{$\begin{array}{l}\text { Motivation to use } \\
\text { Habitual }\end{array}$} & Help in sticking to a routine & $\begin{array}{l}\text { "I think I think that there, there is poten- } \\
\text { tial for the app to to help me keep a more } \\
\text { stable schedule. " } P 83277\end{array}$ \\
\hline & $\begin{array}{l}\text { Lack of other narcolepsy } \\
\text { specific apps }\end{array}$ & $\begin{array}{l}\text { "I have been wanting like an app that } \\
\text { is specific to the needs of people with } \\
\text { narcolepsy for a while." } P 04782\end{array}$ \\
\hline \multirow[t]{2}{*}{$\begin{array}{l}\text { Challenges due to } \\
\text { narcolepsy }\end{array}$} & Memory loss & $\begin{array}{l}\text { "if I remember to do it, I would it just } \\
\text { it was more just a matter of forgetting. " } \\
P 04782\end{array}$ \\
\hline & Social stigma & $\begin{array}{l}\text { "the only time you ever see it or hear } \\
\text { about it is when it's, you know, being } \\
\text { used as a joke in movies or on TV" } \\
P 04782\end{array}$ \\
\hline
\end{tabular}

positively contributing to empowering people with narcolepsy. A breakdown of the individual answers for each statement can be seen in Figure 2.

The highest rated statements were "I notice more how my symptoms change over time" with 5 participants answering with either "Agree" or "Strongly Agree", and "I feel better understood when I tell my physician/ sleep specialist about my symptoms", with also 5 participants answering with either "Agree" or "Strongly Agree", and "I am better able to plan my day in advance because I can make predictions of how I will fell that day". These results indicate that the participants felt that Habitual supported them in understanding their symptoms, which enabled them to have more fruitful discussions with their health professional and also to make predictions about their day. The lowest rated statements was "I am able to have a productive day because I can better anticipate when my energy levels will be high enough" with 6 participants answering either"'Disagree" or "Strongly Disagree".

In order to conduct a more in-depth analysis, we asked if the participants would like to be interviewed. In total, three participants were interviewed and one provided answered our questions in a written format. During the interviews, participants were asked whether Habitual has had any effect on how empowered they feel about managing their condition. All participants said that Habitual has had some impact on their feeling of empowerment. In line with the results from the survey, the most commonly mentioned effect was increased symptom awareness. One interviewee said, "It really made me think about things 


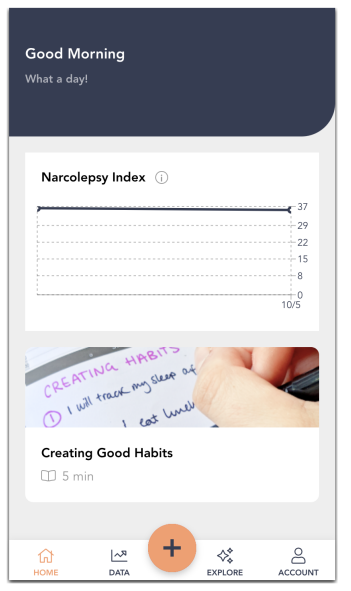

(a) Home screen of the Habitual app, showing an index of narcolepsy management based on the tracked data points, as well as a suggested article

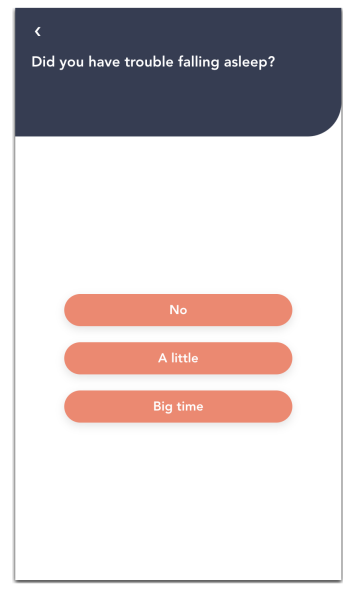

(b) Example of question asked during the morning set of questions, to assess the quality of sleep of the previous night

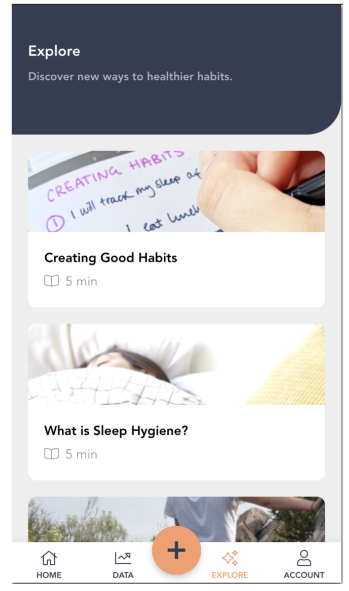

(c) Explore section of the app, which contains educational content about the behavioural management of narcolepsy

Figure 1. Screenshots of the Habitual prototype app used in the 30-day study

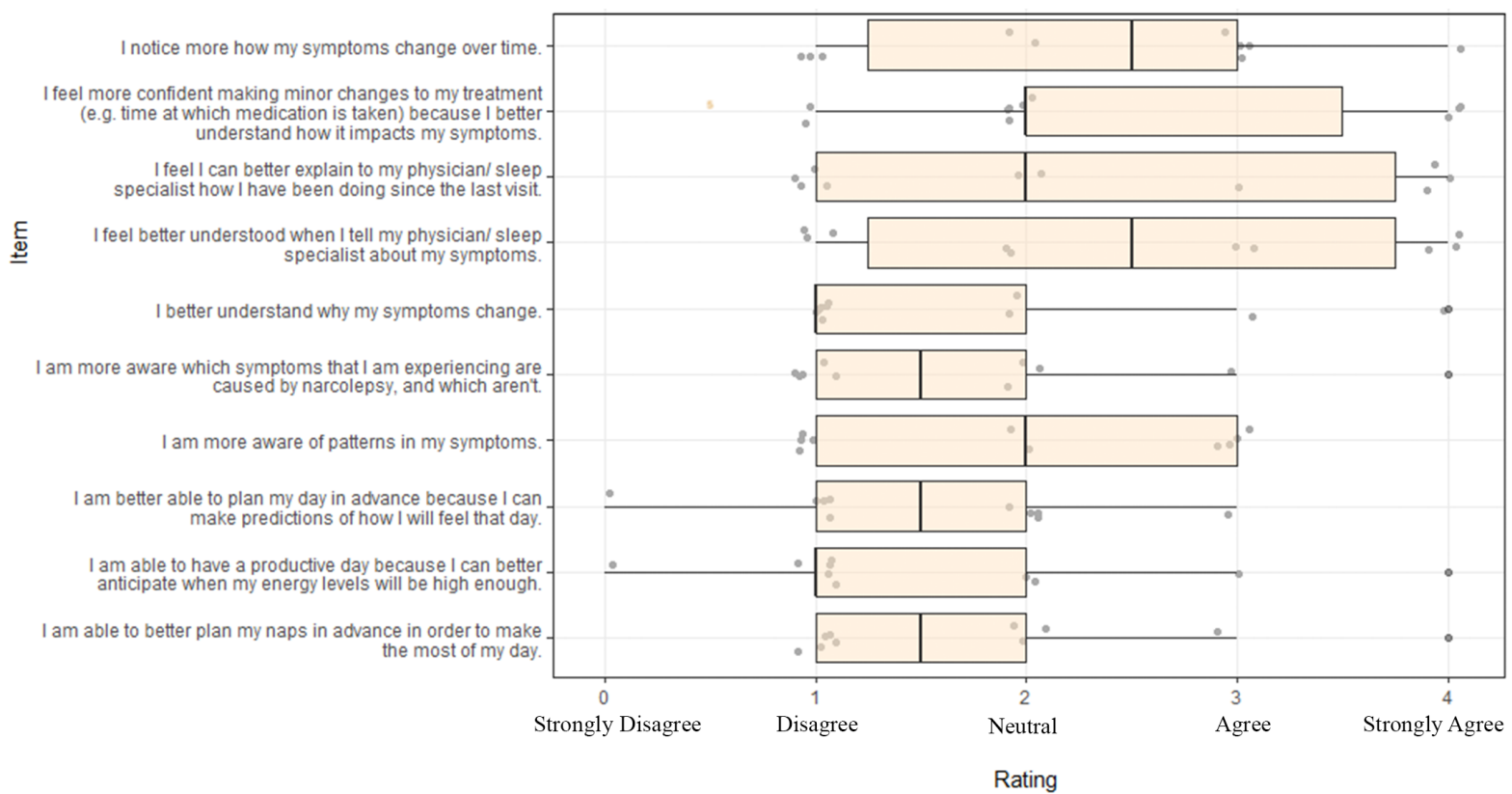

Figure 2. Results of the empowerment survey

that I hadn't" (P83277), while another interviewee explained further why this is important "we're not necessarily paying attention to those smaller pieces that build up to that bigger picture" (P04782). It also helped with correlating and understanding symptoms, for example "being able to see, like on the app that I was typically feeling a little better on days when I got up earlier and took my medicine earlier." (P83277). Other benefits of using the app included mental health support, as one interviewee stated "it helped me to not feel crazy" (P83277), and also a boost of confidence, as an interviewee mentioned "And it gave me a lot of confidence because I don't know anybody that has narcolepsy, and sort of be able to track it myself and see that, that they do have these symptoms, and not just that, but to be able to show it to other people. (P30922)". Many participants felt that communicating symptoms to friends and family facilitates the conversation with physicians and sleep specialists, as one interviewee mentioned "it helps us have a much better conversation with our doctors", as many felt that they often have to advocate for themselves, and one participant expressed that the app "could have actually helped me advocate a little bit more" (P30922). 
Overall, the effects of Habitual on the perception of empowerment of the participants were: support in communicating symptoms and increase symptom awareness. In order to understand how Habitual could be improved, participants were also asked to answer suggestions for future functionalities.

\subsubsection{Suggestions for future functionality}

During the interviews, participants suggested multiple functionalities which would improve their quality of life and impact their feeling of empowerment. This included suggestions on how to improve existing features as well as new functionalities which could be added to the app.

The most commonly mentioned functionality currently not included in the app is a notification or reminder function. Our results suggest that, it is fundamental to remind users to enter the app due to the participants' memory problems. As one interviewee mentioned, "So as with most narcoleptic, folks, we have memory issues. There's a cognitive function"(P04782). Two participants mentioned that the reminder could be in the form of encouragement, explaining that tracking of symptoms is important for the management of narcolepsy, and for advocating for oneself during doctor appointments. Many participants highlighted the importance of anchoring the use of the app with their existing habits.

Another feedback referred to the current tracking and visualization available in the app. Some users felt that the answer range of questions, e.g. sliders, or predefined multiple-choice answers were too broad to capture what was really going on in their lives. As for the visualizations, people wished for a higher level of analysis to fully understand what the symptoms mean and how they correlate. One participant also suggested adding seasonal variables to the analysis, "I think it would be really cool to be able to see day to day, but then to see what your month looks like, and to see how the pattern changes, when the days get shorter" (P83277). Participants also felt that the questions should be personalized to focus more on their specific lifestyle (e.g. driving frequency) and specific symptoms (e.g cataplexy). The feedback also included the wish for personalizing of the data captured in the app, so that the app is only "asking questions about the symptoms that are especially pertinent to each person's experience", as well as a free text area which could act as a placeholder for notes and diary entries. Moreover, participants would like to see a way to easily share data with physician, and also a collection of verified information on topics such as getting through the work day, as well as resources regarding what are their rights as employees and as students. Overall, our findings suggest that when planning mHealth interventions for narcolepsy patients, it is important to provide an application that contains features of: notification/reminders, anchoring it with existing habits, visualization, personalization.

\subsubsection{Motivation to use Habitual}

When asked about their usage of Habitual during the 30 day period, the interviewees disclosed some of their reasons for using habitual that motivated them to continue using it, as well as strategies for ensuring consistent use of the app.Some participants felt that the use of the app was helpful for them to stick to a routine, as one interviewee mentioned, "I think I think that there, there is potential for the app to help me keep a more stable daily routine" (P83277), Similarly, another interviewee pointed out, "I think that there, there is potential for the app to to help me keep a more stable schedule" (P04782). It was also mentioned that it helped them to realize which tasks need to be prioritized in order to make the most of their energy. Participants felt optimistic about the use of the app, as they feel there is currently no app on the market which addresses their needs. One interviewee mentioned, "I have been wanting like an app that is specific to the needs of people with narcolepsy for a while." (P83277) and "I don't feel like there are a whole lot of things that are tailored to our needs" (P04782). Finally, participants mentioned that they were most successful with remembering to use the app on days when they anchored the use of the app with other routine parts of their day. According to one interviewee, "I tried to anchor my logging all the stuff from waking up to when I took my first dose of medicine during the day" (P83277). The participants also mentioned that the usage of the app decreased on days when they did not stick to their daily routine.

\subsubsection{Challenges due to narcolepsy}

This category refers to various challenges mentioned during the interviews that people with narcolepsy have to face because of their condition, independent of the Habitual app. Although these do not directly relate to Habitual, they provide insights into challenges that future digital narcolepsy interventions can address. The most commonly mentioned challenge faced by the participants were memory issues, which 
impacted many aspects of the day, including the use of the app, as many participants would forget to enter their symptoms. One participant said they wanted to track symptoms in the app every day and "if [she] remembered to do it, [she] would it just it was more a matter of forgetting" (P30922). One participant mentioned that forgetfulness impacts their treatment adherence, "But, so if I get knocked off of my routine, I tend to forget everything, including like taking my medicine and stufflike that" (P83277). Other cognitive issues were also mentioned, "a lot of us have serious cognitive function issues. And so the easier it looks or the simpler it looks, the easier it is for the brain for retired people" (P04782). Other commonly mentioned challenges related to the poor lack of awareness of narcolepsy, which also results in social stigma. One participant also mentioned the lack of representation in the media "the only time you ever see [narcolepsy] or hear about it is when it's being used as a joke in movies or on TV" (P83277). Overall, the challenges faced by PWN are memory loss and lack of public awareness.

\section{DISCUSSION}

This study conducted a pilot study to investigate whether a 30-day use of Habitual — a symptom tracking app for people with narcolepsy - has an impact on how participants understand their condition and on their sense of empowerment about its management (RQ1). The study further aimed to identify any additional features that should be considered when designing mHealth interventions for people with narcolepsy (RQ2). To answer RQ1, $10 \mathrm{PwN}$ used Habitual over a 30-day period. In order to evaluate the use of the app, constructs from technology acceptance models were considered, such as, in particular, the perception of usefulness (Davis, 1989), hedonic motivation, and habit (Venkatesh et al., 2012). After the 30-day period, participants were asked to answer a survey aimed at identifying whether their perception of empowerment over the disease had increased. Three patient measurement constructs were used for the survey design: knowledge acquisition, gaining control, and sharing and capacity building (Cerezo et al., 2016). Our results indicate that Habitual had the most impact on participants in terms of three items categorized in the three aforementioned patient measurement constructs. First, regarding knowledge acquisition, after using Habitual for 30 days, most participants claimed to have developed a greater awareness of how their symptoms vary over time. As a consequence, it gave them the power to have more control over their day (gaining control), since most of the participants stated that after using Habitual, they are better able to plan their day in advance because they can make better predictions of how they will feel that day. Finally, in line with the patient measurement construct of sharing and capacity building, most of the participants felt better understood when telling their physician/ sleep specialist about their symptoms.

To answer RQ2, interviews were conducted to gather a more in-depth analysis. As a result, our findings suggest that when planning mHealth interventions for narcolepsy patients, it is fundamental to consider that most of them face challenges related to memory loss, therefore, this study recommends that the application should provide the user with a reminder/notification function. In addition, it seems to be fundamental to anchor the app to the users' existing habits with a view to reminding them to use the app. Our findings also suggest that it is important to allow the user to personalize the application based on their specific symptoms and daily habits.

\section{CONCLUSION}

This paper set out to investigate whether an app for tracking symptoms, daily habits and medication adherence for people with narcolepsy could increase their sense of empowerment. Although using the app for only 30 days provides a very limited understanding of the impact of Habitual, this study offers a positive indication for future mHealth solutions for the management of narcolepsy.

Despite the promising outlook of the study that digital interventions can play an important role in the management of narcolepsy, this pilot-study was conducted with only a small sample $(n=10)$. Considering that narcolepsy is a rare disease and patients face severe cognitive difficulties, studies on mHealth development for narcolepsy tend to use small samples (Quaedackers et al., 2020). Therefore, largerscale studies are required to verify our findings. Another limitation of the study is the selection bias of participants. The participants registered to take part in this study after reading about it on the Wake Up Narcolepsy website or social media channels. It is therefore possible that those who applied to take part already felt positive about using an app to track narcolepsy-related aspects. Future studies should test the openness to using 
an app for the management of narcolepsy with a wider cohort. Moreover, researchers could investigate the use of the app beyond 30 days and set up control groups and statistical analysis to compare results from before and after using the intervention. Finally, future studies should also identify acceptance criteria. Nevertheless, the contribution of this research is twofold. First, this study contributes to the body of knowledge of technology acceptance models and user-centred design for mHealth interventions by presenting relevant constructs that should inform the design and creation of mHealth interventions for people with narcolepsy. Secondly, we contribute to practice by translating the needs of the participants

\section{ACKNOWLEDGMENTS}

We would like to thank Wake Up Narcolepsy for their input during the development of the app, as well as their great support with recruiting participants for this study. We would also like to thank Takeda Pharmaceutical Company for their input during the project.

\section{REFERENCES}

Acosta Salgado, L., Bonjour, E., \& Rakotondranaivo, A. (2020). Towards an improved acceptability assessment model of innovative solutions in the design phase. Int. J. Business Information Systems, 10(Y4), 000-000.

Cerezo, P. G., Juvé-Udina, M.-E., \& Delgado-Hito, P. (2016). Concepts and measures of patient empowerment: A comprehensive review. Revista da Escola de Enfermagem da USP, 50(4), 667-674.

Dauvilliers, Y., Arnulf, I., \& Mignot, E. (2007). Narcolepsy with cataplexy. The Lancet, 369(9560), 499-511.

Davis, F. D. (1989). Perceived usefulness, perceived ease of use, and user acceptance of information technology. MIS Quarterly, 13(3), 319.

De Paula, D., Zarske Bueno, J., \& Viljoen, A. (2021). Defining archetypes and requirements for mhealth interventions in rural kenya: An investigation in collaboration with curafa ${ }^{\mathrm{TM}}$, In Proceedings of the 54th hawaii international conference on system sciences.

Gioia, D. A., Corley, K. G., \& Hamilton, A. L. (2012). Seeking qualitative rigor in inductive research. Organizational Research Methods, 16(1), 15-31.

Holmström, I., \& Röing, M. (2010). The relation between patient-centeredness and patient empowerment: A discussion on concepts. Patient Education and Counseling, 79(2), 167-172.

Kallweit, U., Schmidt, M., \& Bassetti, C. L. (2017). Patient-reported measures of narcolepsy: The need for better assessment. Journal of Clinical Sleep Medicine, 13(05), 737-744.

Quaedackers, L., Wit, J. D., Pillen, S., Gilst, M. V., Batalas, N., Lammers, G. J., Markopoulos, P., \& Overeem, S. (2020). A mobile app for longterm monitoring of narcolepsy symptoms: Design, development, and evaluation. JMIR mHealth and uHealth, 8(1), e14939.

Raggi, A., Plazzi, G., \& Ferri, R. (2019). Health-related quality of life in patients with narcolepsy. The Journal of Nervous and Mental Disease, 207(2), 84-99.

Rowland, S. P., Fitzgerald, J. E., Holme, T., Powell, J., \& McGregor, A. (2020). What is the clinical value of mHealth for patients? npj Digital Medicine, 3(1).

Small, N., Bower, P., Chew-Graham, C. A., Whalley, D., \& Protheroe, J. (2013). Patient empowerment in long-term conditions: Development and preliminary testing of a new measure. BMC Health Services Research, 13(1).

Uebernickel, F., Jiang, L., Brenner, W., Pukall, B., Naef, T., \& Schindlholzer, B. (2020). Design thinking: The handbook. World Scientific.

Venkatesh, Thong, \& Xu. (2012). Consumer acceptance and use of information technology: Extending the unified theory of acceptance and use of technology. MIS Quarterly, 36(1), 157.

World Health Organization. (2011). MHealth: New horizons for health through mobile technologies. [OCLC: 759446797]. Geneva, World Health Organization. Retrieved October 12, 2020, from http://www.who.int/goe/publications/goe_mhealth_web.pdf

World Health Organization Regional Office for Europe. (2012). Health 2020 policy framework and strategy document. https://www.euro.who.int/_data/assets/pdf_file/0020/170093/RC62wd08-Eng.pdf 